\title{
THE UNIVALENCE OF FUNCTIONS ASYMPTOTIC TO NONCONSTANT LOGARITHMIC MONOMIALS ${ }^{1}$
}

\author{
E. W. CHAMBERLAIN
}

Introduction. The title refers to analytic functions $s(x)$ which behave like nonconstant logarithmic monomials $M(x)=c x^{m_{0}}(\log x)^{m_{1}}$ $\cdots\left(\log _{r} x\right)^{m_{r}}$ (where $c$ is a complex number $\neq 0$, the $m_{i}$ are real, and $\log _{N}$ is the $N$-fold iterate of the principal determination of $\log$ ) in the sense that $s(x) / M(x) \rightarrow 1$ as $x \rightarrow \infty$ in the complex plane.

Definition. An analytic function $E$ is said to $\rightarrow 0$ rapidly enough for $M$ if $E \rightarrow 0$ and $\left(M / M^{\prime}\right) E^{\prime} \rightarrow 0$ as $x \rightarrow \infty$.

Theorem 2 states that if $s=M(1+E)$ where $E \rightarrow 0$ rapidly enough for $M$, then $s$ is $1-1$ in some neighborhood of infinity.

The neighborhood bases for $\infty$ with which we shall be concerned are families $\bar{F}(\alpha, \beta)$ whose elements are sector-like regions $V(\alpha, \beta, \xi)$ defined as follows: Let $-\pi \leqq \alpha<\beta \leqq \pi$. Let $\xi(\delta)$ be a real-valued function defined and bounded below on some subinterval $(0, \gamma)$ of $(0,(\beta-\alpha) / 2)$. Let $T\left(\alpha+\delta, \beta-\delta, \xi(\delta) e^{i \mu}\right)$ be the sector $\{z: \alpha+\delta$ $\left.<\arg \left(z-\xi(\delta) e^{i \mu}\right)<\beta-\delta\right\}$, where $\mu=(\alpha+\beta) / 2 . V(\alpha, \beta, \xi)$ is then $U\left\{T\left(\alpha+\delta, \beta-\delta, \xi(\delta) e^{i \mu}\right): 0<\delta<\gamma\right\}$. The family of all such $V(\alpha, \beta, \xi)$ is denoted $\bar{F}(\alpha, \beta)$. Such neighborhood bases are dealt with in the asymptotic theory of ordinary differential equations in the complex domain, as developed in [1] and [2]. (See [1, p. 44].)

Remark. Taking $s=M(1+E)$ where $M(x)=\log x$ and $E(x)$ $=x^{i} / \log x$, it is easily seen that $s / M \rightarrow 1$ over $\bar{F}(-\pi,+\pi)$ but $s$ is not univalent on any member of $\bar{F}(-\pi,+\pi)$ (since $s^{\prime}=0$ infinitely far out on the real axis); $E \rightarrow 0$, but not rapidly enough for $M$ in this case.

Theorem 2 justifies a large class of changes of independent variables in the study of differential equations. Functions $f(x)$ (in particular, coefficients of differential equations) can be regarded legitimately as functions $F(s)$, over suitable neighborhoods of $\infty$, on the condition that $s$ be asymptotic to a nonconstant logarithmic monomial $M$ in the sense described. Changes of this type are important in the treatment of linear differential operators with repeated approximate factors (cf. [3] where such a change is effected by the formal substitution $-W(x) d x=d s)$.

Presented to the Society, January 27, 1966; received by the editors May 27, 1965.

1 This research was supported by NSF Grant No. GP-3248. 
Theorem 1 shows that the class of substitutions effected by writing $d s=V(x) d x$ where $V / V_{0} \rightarrow 1, V_{0}$ being a logarithmic monomial such that $\int_{x_{0}}^{\infty} V_{0}=\infty$, is precisely the class of substitutions $s=M(1+E)$, where $E \rightarrow 0$ rapidly enough for $M$ and where $M \rightarrow \infty$ as $x \rightarrow \infty$.

The principal device used in this paper is the Sharp Form of the Generalized Mean Value Theorem, given in Lemma 1. It asserts, roughly, that the tangent vector to a simple differentiable arc attains parallelism (as opposed to antiparallelism) to the vector from initial to terminal point. Consequently, a simple closed curve with no more than two points at which the curve is not smooth has tangent vectors whose arguments differ by $\pi$. The crux of the univalence discussion is that for the functions $s(x)$ and for the domains $V(\alpha, \beta, \xi)$ in question, the images of certain paths joining arbitrary points in $V(\alpha, \beta, \xi)$ are curves on which the argument of the tangent vector is limited to values differing by less than $\pi$-partly because of limitations on $s^{\prime}(x)$, partly because of the geometry of the path. Thus $s$ cannot map such a path onto a closed curve, and hence assumes two different values at the end points.

Notation. $\mu$ will always represent one half the sum of the first two arguments of the function $T$. For complex $z_{1}, z_{2},\left(z_{1}, z_{2}\right)$ and $\left[z_{1}, z_{2}\right]$ will represent the open and closed line segments determined by the $z_{i}$.

Lemma 1 (Sharp Form of the Generalized Mean Value THEOREM). Let $C$ be a simple arc given by a map $z(t)=(x(t), y(t))$ from $[0,1]$ into the complex plane which is continuous and $1-1$ on $[0,1]$ and such that $z^{\prime}(t)$ exists and is never zero for all $t \in(0,1)$. Then there exists a $t_{1} \in(0,1)$ such that $\arg \left(z^{\prime}\left(t_{1}\right)\right)=\arg (z(1)-z(0))$.

Proof. We discuss the case in which $\arg (z(1)-z(0))=0$. No generality is lost in assuming that $C \cap[z(0), z(1)]=\{z(0), z(1)\}$. Defining $z(t)=z(1)+(t-1)(z(0)-z(1))$ for $1<t \leqq 2$, we may further assume that the simple closed curve $\bar{C}$ given by $z=z(t), 0 \leqq t \leqq 2$, is positively oriented. Let $t_{1} \in(0,1) \cup(1,2)$ be such that $y\left(t_{1}\right) \leqq y(t)$ for all $t \in[0,2]$. Clearly $\arg \left(z^{\prime}\left(t_{1}\right)\right)=0$ or $=\pi$. We wish to show that $\arg \left(z^{\prime}\left(t_{1}\right)\right)=0$.

Since $z^{\prime}\left(t_{1}\right)$ is a nonzero real number, we can write $\bar{C}=\Lambda \cup(\bar{C}-\Lambda)$ where $\Lambda$ is a small arc through $z\left(t_{1}\right)$ such that $\Lambda \subset\left\{z: \arg \left(z-z\left(t_{1}\right)\right)\right.$ $\in[0, \pi / 4] \cup[3 \pi / 4, \pi]\} \cup\left\{z\left(t_{1}\right)\right\}$. For $z(t) \in \Lambda$, either

$$
\arg \left(z(t)-z\left(t_{1}\right)\right) \in[0, \pi / 4] \text { for } t<t_{1}
$$

and

$$
\arg \left(z(t)-z\left(t_{1}\right)\right) \in[3 \pi / 4, \pi\rfloor \text { for } t>t_{1},
$$


or else

$$
\left.\arg (z(t))-z\left(t_{1}\right)\right) \in[3 \pi / 4, \pi] \text { for } t<t_{1}
$$

and

$$
\arg \left(z(t)-z\left(t_{1}\right)\right) \in[0, \pi / 4] \text { for } t>t_{1} .
$$

Suppose (a) is the case-i.e., suppose $\arg \left(z^{\prime}\left(t_{1}\right)\right)=\pi$. Let $q=(X, Y)$ be a point such that $X=x\left(t_{1}\right), Y>y\left(t_{1}\right)$, and sufficiently near $z\left(t_{1}\right)$ that $\{z: \arg (z-q)=-\pi / 2\} \cap \bar{C}=\left\{z\left(t_{1}\right)\right\}$. As $z(t)$ describes $\bar{C}-\Lambda$ from the left end point of $\Lambda$ to the right end point of $\Lambda, \arg (z(t)-q)$ varies from a value near $\pi$ to a value near 0 (for on $\bar{C}-\Lambda$, $\arg (z(t)-q)$ assumes no value congruent to $3 \pi / 2)$. As $z(t)$ describes $\Lambda$ from right to left, $\arg (z(t)-q)$ varies from its value near 0 to a value congruent to its initial value; in so doing, $\arg (z(t)-q)$ passes through a value congruent to $-\pi / 2$ but through no value congruent to $+\pi / 2$. Hence the terminal value of $\arg (z(t)-q)$ equals its initial value minus $2 \pi$. This contradicts the assumption that $\bar{C}$ is positively oriented. Therefore (b) is the case-i.e., $\arg \left(z^{\prime}\left(t_{1}\right)\right)=0$; and $z\left(t_{1}\right) \in C$ since $\arg \left(z^{\prime}(t)\right)$ $=\pi$ for $1<t<2$.

(I am grateful to Julius S. Dwork for useful suggestions incorporated in this proof.)

Lemma 2. Let $z(t)$ map the interval $[a, b]$ continuously into the plane in such $a$ way that $a \leqq t^{\prime}<t^{\prime \prime} \leqq b$, then $z\left(t^{\prime}\right)=z\left(t^{\prime \prime}\right)$ if and only if $t^{\prime}=a$ and $t^{\prime \prime}=b$; and suppose that for some $c \in(a, b), z^{\prime}(t)$ exists and is never zero on $(a, c) \cup(c, b)$. Then there exist $t_{1} \in(a, c)$ and $t_{2} \in(c, b)$ such that $\left|\arg \left(z^{\prime}\left(t_{1}\right)\right)-\arg \left(z^{\prime}\left(t_{2}\right)\right)\right|=\pi$.

Proof. Apply Lemma 1 to the two simple arcs obtained by restricting $z$ to the intervals $[a, c]$ and $[c, b]$.

Lemma 3. Let $E$ be analytic in $V \in \bar{F}(\alpha, \beta)$ and let $E \rightarrow 0$ over $\bar{F}(\alpha, \beta)$. Let $F$ be analytic in $V$ and such that $F / W \rightarrow 1$ over $\bar{F}(\alpha, \beta)$, where $W(x)$ $=c x^{-1}(\log x)^{-1} \cdots\left(\log _{k} x\right)^{-1+\tau}\left(\log _{k+1} x\right)^{a_{1}} \ldots\left(\log _{k+r} x\right)^{a_{r}}$ with $c \neq 0$ and $\tau>0$. Let $x_{0} \in V$. Then $\int_{x_{0}}^{x} E F / \int_{x_{0}}^{x} F \rightarrow 0$ over $\bar{F}(\alpha, \beta)$.

Proof. First we establish

Assertion A. Let $V_{1}, B$ be such that $V_{1} \in \bar{F}(\alpha, \beta)$ and $|E(z)|<B$ for all $z \in V_{1}$. Let $x(r)=r e^{i \mu}$ where $\mu=(\alpha+\beta) / 2$. Let $\delta \in(0,(\beta-\alpha) / 2)$. Then for all sufficiently large $r$ there exists a positive number $S(r, \delta)$ such that $\left|\int_{x(r)}^{x} E F / \int_{x(r)}^{x} F\right|<4 B$ whenever $|x|>S(r, \delta)$ and $x \in T(\alpha+\delta, \beta-\delta, x(r))$.

Proof of Assertion A. Let $R$ be a positive number so large that $V_{1} \supset T(\alpha+\delta, \beta-\delta, x(R)), F$ is analytic on the closure of $T(\alpha+\delta$, 
$\beta-\delta, x(R))$, and $|F(x)|>\frac{1}{2} \tau|x|^{-1}(\log |x|)^{-1} \cdots\left(\log _{k}|x|\right)^{-1+\tau / 2}$ for all $x \in T(\alpha+\delta, \beta-\delta, x(R))$. We shall show that for each $r \geqq R$ there exists an $S(r, \delta)$ with the prescribed property.

Let $r \geqq R$. It is easily seen that there exists a positive number $S_{1} \geqq x(r)$ such that for each $\phi \in[\alpha+\delta, \beta-\delta]$ there exists a constant $c(\phi)$ such that $|\arg (F(x))-c(\phi)|<\frac{1}{2}$ whenever $|x| \geqq S_{1}$ and $\arg (x-x(r))=\phi$. Then for each $x$ such that $|x|>S_{1}$ and $\alpha+\delta$ $<\arg (x-x(r))<\beta-\delta$, if we let $\left\{x_{1}\right\}=[x, x(r)] \cap\left\{z:|z|=S_{1}\right\}$ and integrate along $\left[x_{1}, x\right]$, we obtain

$$
\begin{aligned}
& \left|\int_{x_{1}}^{x} F(t) d t\right| \\
& \quad=\left|\int_{x_{1}}^{x}\right| F(t)|\exp [i c(\phi)+i \arg (d t)+i(\arg (F(t))-c(\phi))]| d t|| \\
& \quad \geqq \int_{x_{1}}^{x}|F(t)| \cos (\arg (F(t)-c(\phi)))|d t|>(7 / 8) \int_{x_{1}}^{x}|F(t)||d t| \\
& \quad>(7 / 8)\left(\left(\log _{k}|x|\right)^{\tau / 2}-\left(\log _{k}\left|x_{1}\right|\right)^{\tau / 2}\right) .
\end{aligned}
$$

Let $B_{1}$ be an upper bound for $\left\{\int_{x(r)}^{x}|F(t)||d t|: x \in\left\{z:|z|=S_{1}\right\}\right.$ $\cap T(\alpha+\delta, \beta-\delta, x(r))$, integrated over $[x(r), x]\}$. Let $S_{2}$ be a positive number so large that $\left(\log _{k} S_{2}\right)^{\tau / 2}-\left(\log _{k} S_{1}\right)^{\tau / 2}>2 B_{1}$. Then if $x$ is any member of $T(\alpha+\delta, \beta-\delta, x(r))$ such that $|x| \geqq S_{2}$, and $x_{1}=[x(r), x]$ $\cap\{z:|z|=S\}$, we have

$$
\begin{aligned}
\left|\int_{x(r)}^{x} E F\right| \leqq B\left(\int_{x(r)}^{x_{1}}|F||d t|+\int_{x_{1}}^{x}|F||d t|\right) \\
\quad=B \int_{x_{1}}^{x}|F||d t| \cdot\left(1+\left(\int_{x(r)}^{x_{1}}|F||d t|\right) /\left(\int_{x_{1}}^{x}|F||d t|\right)\right) \\
\quad \leqq(3 B / 2) \int_{x_{1}}^{x}|F||d t| .
\end{aligned}
$$

At the same time,

$$
\left|\int_{x(r)}^{x_{1}} F(t) d t\right| \leqq \int_{x(r)}^{x_{1}}|F||d t| \leqq B_{1}
$$

and

$$
\left|\int_{x_{1}}^{x} F(t) d t\right|>(7 / 8) \int_{x_{1}}^{x}|F||d t|>(7 / 8) \cdot 2 B_{1}
$$




$$
\begin{aligned}
\left|\int_{x(r)}^{x} F(t) d t\right| & =\left|\int_{x_{1}}^{x} F(t) d t\right| \cdot\left|1+\left(\int_{x(r)}^{x_{1}} F(t) d t\right) /\left(\int_{x_{1}}^{x} F(t) d t\right)\right| \\
& >(7 / 8)\left(\int_{x_{1}}^{x}|F||d t|\right)\left(1-B_{1} /\left((7 / 8) 2 B_{1}\right)\right) .
\end{aligned}
$$

Hence $\left|\int_{x(r)}^{x} E F / \int_{x(r)}^{x} F\right|<4 B$ whenever $|x| \geqq S(r, \delta)$ if we define $S(r, \delta)=S_{2}$.

Assertion B. Let $x_{00}=r_{0} e^{i \mu} \in V$. Then for each $\epsilon>0$ and each $\delta$ in $(0,(\beta-\alpha) / 2)$ there exists a sector $T\left(\alpha+\delta, \beta-\delta, r e^{i \mu}\right)$ such that $\left|\int_{x_{00}}^{x} E F / \int_{x_{00}}^{x} F\right|<\epsilon$ for every $x \in T\left(\alpha+\delta, \beta-\delta, r e^{i \mu}\right)$.

Proof of Assertion B. Let $\epsilon>0$ and $\delta \in(0,(\beta-\alpha) / 2)$. Since there exists an element of $\bar{F}(\alpha, \beta)$ in which $|E|<\epsilon / 16$, Assertion A implies the existence of positive numbers $r$ and $S$ such that $\left|\int_{x(r)}^{x} E F / \int_{x(r)}^{x} F\right|<\epsilon / 4$ for each $x \in T(\alpha+\delta, \beta-\delta, x(r)) \cap\{z:|z|>S\}$. We have $\left|\int_{x_{00}}^{x} E F / \int_{x_{00}}^{x} F\right| \leqq N_{1} / N_{0}+N_{2} / N_{0}$ where $N_{0}=\left|\int_{x(r)}^{x} F\right|$ $\left|1+\left(\int_{x_{00}}^{x(r)} F / \int_{x(r)}^{x} F\right)\right|, N_{1}=\left|\int_{x_{00}}^{x(r)} E F\right|$, and $N_{2}=\left|\int_{x(r)}^{x} E F\right|$. Let $S^{\prime} \geqq S$ be so large that whenever $x \in T^{\prime}=T(\alpha+\delta, \beta-\delta, x(r)) \cap\left\{z:|z|>S^{\prime}\right\}$ the following inequalities hold: (i) $\left|1+\left(\int_{x_{00}}^{x(r)} F / \int_{x(r)}^{x} F\right)\right|>\frac{1}{2}$, (ii) $\left|\int_{x_{00}}^{x(r)} E F / \int_{x(r)}^{x} F\right|<\epsilon / 4$. Let $\bar{r}$ be so large that $T\left(\alpha+\delta, \beta-\delta, \bar{r} e^{i \mu}\right) \subset T^{\prime}$. Then whenever $x \in T\left(\alpha+\delta, \beta-\delta, \quad \bar{r} e^{i \mu}\right)$ we have $\left|\int_{x_{00}}^{x} E F / \int_{x_{00}}^{x} F\right|$ $<2(\epsilon / 4)+2(\epsilon / 4)=\epsilon$.

Assertion B establishes Lemma 3 for $x_{0}$ for the special form $x_{00}$; to extend the result to arbitrary $x_{0} \in V$, write $\int_{x_{0}}^{x} E F / \int_{x_{0}}^{x} F$

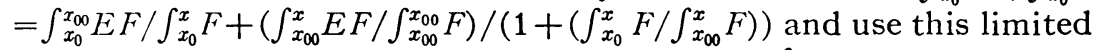
form of Assertion B together with the fact that $\int_{x_{0}}^{x} F \rightarrow \infty$.

Theorem 1. Let $M(x)=c x^{m_{0}}(\log x)^{m_{1}} \cdots\left(\log _{r} x\right)^{m_{r}}$ be a nonconstant logarithmic monomial, with $m_{k}>0$ and $m_{i}=0$ for $i<k$. Let $W$ be the logarithmic monomial such that $M^{\prime} / W \rightarrow 1$. Then (a) if $E \rightarrow 0$ rapidly enough for $M, M(1+E)$ can be expressed as an indefinite integral: $M(x)(1+E(x))=M\left(x_{0}\right)\left(1+E\left(x_{0}\right)\right)+\int_{x_{0}}^{x} W\left(1+E_{0}\right)$, where $E_{0} \rightarrow 0 ;$ and (b) if $E_{0}$ is any analytic function which $\rightarrow 0$, then $\int_{x_{0}}^{x} W\left(1+E_{0}\right)$ $=M(1+E)$ where $E \rightarrow 0$ rapidly enough for $M$.

Proof. (a) We have $M(x)(1+E(x))=M\left(x_{0}\right)\left(1+E\left(x_{0}\right)\right)$ $+\int_{x_{0}}^{x} M^{\prime}\left(1+E+\left(M / M^{\prime}\right) E^{\prime}\right)$; since $E \rightarrow 0$ rapidly enough for $M$, it follows easily that the integrand can be expressed in the form $W\left(1+E_{0}\right)$.

(b) $W\left(1+E_{0}\right)=M^{\prime}\left(1+E_{1}\right)$ where $E_{1} \rightarrow 0$. Therefore $\int_{x_{0}}^{x} W\left(1+E_{0}\right)$ $=M(x)\left[\left(\int_{x_{0}}^{x} M^{\prime} / M(x)\right)+\left(\int_{x_{0}}^{x} E_{1} M^{\prime} / M(x)\right)\right]$ where the first term in the bracket obviously $\rightarrow 1$ while the second term $\rightarrow 0$ by Lemma 3 . Thus $\int_{x_{0}}^{x} W\left(1+E_{0}\right)=M(x)(1+E(x))$ with $E \rightarrow 0$. We have $E^{\prime}=A+B$ where $A=\left(M M^{\prime}-M^{\prime} \int_{x_{0}}^{x} M^{\prime}\right) / M^{2}$ and $B=\left(M E_{1} M^{\prime}-M^{\prime} \int_{x_{0}}^{x} E_{1} M^{\prime}\right) / M^{2}$. To 
show that $E \rightarrow 0$ rapidly enough for $M$, we have $\left(M / M^{\prime}\right) A=1$ $-\left(\int_{x_{0}}^{x} M^{\prime} / M(x)\right) \rightarrow 0$ and $\left(M / M^{\prime}\right) B=E_{1}-\left(\int_{x_{0}}^{x} E_{1} M^{\prime} / M(x)\right)$, the latter tending to 0 by Lemma 3 .

THEOREM 2. Let $M(x)$ be a nonconstant logarithmic monomial, as above, with $m_{k}$ the first nonzero member of $\left(m_{0}, \cdots, m_{r}\right)$. Let $E$ be analytic and let $E \rightarrow 0$ rapidly enough for $M$ over $\bar{F}(\alpha, \beta)$. Let $\left(\alpha^{\prime}, \beta^{\prime}\right) \subset(\alpha, \beta)$ and $\left|m_{0}\left(\beta^{\prime}-\alpha^{\prime}\right)\right| \leqq 2 \pi$. Then $M(1+E)$ is univalent in some member of $\bar{F}\left(\alpha^{\prime}, \beta^{\prime}\right)$.

Proof. We shall restrict our attention to the case where $m_{k}>0$, for it is readily seen that if $m_{k}<0$ then $M(1+E)=\left(M_{1}\left(1+E_{1}\right)\right)^{-1}$ where the first nonzero exponent in $M_{1}$ is positive and $E_{1}$ is analytic and $E_{1} \rightarrow 0$ rapidly enough for $M_{1}$ over $\bar{F}(\alpha, \beta)$.

Expressing $M(1+E)$ as an indefinite integral as in Theorem 1, we shall prove that $s(x)=\int_{x_{0}}^{x} W\left(1+E_{0}\right)$ is univalent in some member of $\bar{F}\left(\alpha^{\prime}, \beta^{\prime}\right)$. The following cases will be discussed in detail:

Case 1. $\beta-\alpha \leqq \pi ; k>0$, or $k=0$ and $0<m_{k} \leqq 1$;

Case 2. $\beta-\alpha>\pi ; k>0$, or $k=0$ and $0<m_{k} \leqq 1$. The remaining case, in which $k=0$ and $m_{0}>1$, may be treated similarly, but the details are more complicated. We shall omit this complicated treatment and dispose of this case as follows: Write $M(1+E)=[\tilde{M}(1+\widetilde{E})]^{m 0}$ where $\tilde{M}(x)=c^{1 / m_{0}} x^{1}(\log x)^{m_{1} / m_{0}} \cdots\left(\log _{r} x\right)^{m_{r} / m_{0}} \quad$ and $\quad \tilde{E}=(1+E)^{1 / m_{0}}-1$. Restricting $x$, from the outset, to a member of $\bar{F}(\alpha, \beta)$ in which $|E|$ $<1$ and defining $(1+E)^{1 / m_{0}}=\exp \left(\left(1 / m_{0}\right) \log (1+E)\right)$ (using the principal value of $\log$ ), we have $\tilde{E} \rightarrow 0$ over $\bar{F}(\alpha, \beta)$. $\tilde{E} \rightarrow 0$ rapidly enough for $\tilde{M}$, i.e. $x \tilde{E}^{\prime} \rightarrow 0$, automatically in this case, by Lemma 4 . By the validity of the present theorem in Cases 1 and $2, \widetilde{M}(1+\tilde{E})$ is univalent in a member of $\bar{F}(\alpha, \beta)$, hence in a member of each $\bar{F}\left(\alpha^{\prime}, \beta^{\prime}\right)$. It remains only to show that $\tilde{M}(1+\tilde{E})$ maps a member of each $\bar{F}\left(\alpha^{\prime}, \beta^{\prime}\right)$ into a region in which $z \rightarrow z^{m_{0}}$ is univalent, and this is done in Lemma 5.

For Cases 1 and 2 we construct an element $V(\alpha, \beta, \xi)$ of $\bar{F}(\alpha, \beta)$ in which $s$ is univalent by defining the function $\xi(\delta)$ as follows: For a suitable subinterval $(0, \gamma)$ of $(0,(\beta-\alpha) / 2)$, and for each $\delta \in(0, \gamma)$, $\xi(\delta)$ is chosen to be a positive number so large that for all $x \in T_{\delta}$ $=T\left(\alpha+\delta, \beta-\delta, \xi(\delta) e^{i \mu}\right)$,

$$
\left|\arg \left(x^{1-m_{0}} W(x)\left(1+E_{0}(x)\right)\right)-\arg (c)\right|<\delta / 4 .
$$

(This is possible because of obvious properties of iterated logarithms and because $E_{0} \rightarrow 0$ over $\bar{F}(\alpha, \beta)$.) Then $s$ is shown to be univalent in $V(\alpha, \beta, \xi)$ by applying Lemma 2 as follows: For each pair $x_{1}, x_{2}$ in $V(\alpha, \beta, \xi)$ we construct a map $x(t), 0 \leqq t \leqq 2$, by choosing a third 
point $x_{3}$ in a manner depending on circumstances and defining $x(t)$ $=x_{1}+t\left(x_{3}-x_{1}\right)$ for $0 \leqq t \leqq 1$ and $x(t)=x_{3}+(t-1)\left(x_{2}-x_{3}\right)$ for $1<t \leqq 2$. We define $z(t)=s(x(t))$, and $F\left(t_{1}, t_{2}\right)=\arg \left(z^{\prime}\left(t_{1}\right)\right)-\arg \left(z^{\prime}\left(t_{2}\right)\right)$ for $\left(t_{1}, t_{2}\right) \in J \times J$ where $J=(0,1) \cup(1,2)$. Then we show that $|F|<\pi$ on $J \times J$. Lemma 2 implies that $z(t)$ maps no subinterval of $[0,2]$ onto a simple closed curve, whence it follows that $s\left(x_{1}\right) \neq s\left(x_{2}\right)$.

Since $\left|F\left(t_{1}, t_{2}\right)\right|$ is symmetric in $t_{1}$ and $t_{2}$, we shall only consider pairs $\left(t_{1}, t_{2}\right) \in J \times J$ such that $t_{1}<t_{2}$.

Notation. (1) Let $\nu=m_{0}-1$.

(2) Let $F_{1}=F_{1}\left(t_{1}, t_{2}\right)=\nu\left(\arg \left(x\left(t_{1}\right)\right)-\arg \left(x\left(t_{2}\right)\right)\right)$.

(3) Let $F_{2}=F_{2}\left(t_{1}, t_{2}\right)=\arg \left(x^{\prime}\left(t_{1}\right)\right)-\arg \left(x^{\prime}\left(t_{2}\right)\right)$.

(4) Let $F_{3}=F_{3}\left(t_{1}, t_{2}\right)=A_{1}-A_{2}$, where $A_{i}=\arg \left[\left(x\left(t_{i}\right)\right)^{-\triangleright} W\left(x\left(t_{i}\right)\right)(1\right.$ $\left.\left.+E_{0}\left(x\left(t_{i}\right)\right)\right)\right]-\arg (c)$, so that $F=F_{1}+F_{2}+F_{3}$.

Case 1. Consider $\xi(\delta)$ to be defined for $0<\delta<(\beta-\alpha) / 2$. Let $x_{1}$ and $x_{2} \in V(\alpha, \beta, \xi)$; let $\delta_{1}, \delta_{2}$ be such that $x_{i} \in T_{\delta_{i}}, i=1,2$.

(1a) Suppose $\left[x_{1}, x_{2}\right] \subset T_{\delta_{1}} \cup T_{\delta_{2}}$. Let $x_{3} \in\left(x_{1}, x_{2}\right)$. Then if $t_{1}$ and $t_{2}$ are such that $\left\{x\left(t_{1}\right), x\left(t_{2}\right)\right\} \subset T_{\delta_{j}}$ for $j=1$ or $j=2$, we have $\left|F_{1}\right|<\beta-\alpha$ $-2 \delta_{j}, F_{2}=0$, and $\left|F_{3}\right|<\delta_{j} / 2$, so $|F|<\pi$. For any other $\left(t_{1}, t_{2}\right) \in J \times J$ we have $\left|F_{1}\right|<\beta-\alpha-\delta_{1}-\delta_{2}, \quad F_{2}=0$, and $\left|F_{3}\right|<\delta_{1} / 4+\delta_{2} / 4$; thus $|F|<\pi$ on $J \times J$.

(1b) In the contrary subcase we may suppose $x_{1} \in T_{\delta_{1}}-T_{\delta_{2}}$ while $x_{2} \in T_{\delta_{2}}-T_{\delta_{1}}, \delta_{1}>\delta_{2}$, and $\arg \left(x_{1}\right)>\arg \left(x_{2}\right)$. (The other possibilities lead to similar discussions.) In this situation there exists a point $x_{3} \in T_{\delta_{1}} \cap T_{\delta_{2}}$ such that $\arg \left(x_{3}-x_{1}\right)=\alpha+\delta_{1}$ and $\arg \left(x_{2}-x_{3}\right)=\alpha+\delta_{2}$. From (1a) we see that $|F|<\pi$ on $(0,1) \times(0,1) \cup(1,2) \times(1,2)$. For $t_{1}<1<t_{2}$ we have $0 \geqq F_{1}>\left(\alpha+\delta_{2}\right)-\left(\beta-\delta_{1}\right), \quad F_{2}=\delta_{1}-\delta_{2}$, and $\left|F_{3}\right|$ $<\delta_{1} / 4+\delta_{2} / 4<\delta_{1} / 2$, so $\delta_{1}>\delta_{1}-\delta_{2} \geqq F_{1}+F_{2}>-(\beta-\alpha)+2 \delta_{1}$, whence $\pi>3 \delta_{1} / 2>F>-(\beta-\alpha)+3 \delta_{1} / 2>-\pi$, so $|F|<\pi$ on $J \times J$.

Case 2. Consider $\xi(\delta)$ to be defined on $(0, \gamma) \subset(0,(\beta-\alpha-\pi) / 2)$. Let $x_{i} \in T_{\delta_{i}}, i=1,2$, where $\left\{\delta_{1}, \delta_{2}\right\} \subset(0, \gamma)$.

(2a) Suppose $\arg \left(x_{1}\right)=\arg \left(x_{2}\right)$. Take $x_{3} \in\left(x_{1}, x_{2}\right)$. Then $F_{1}=F_{2}$ $=0$ and $\left|F_{3}\right|<\max \left(\delta_{1} / 2, \delta_{2} / 2\right)<\pi$, so $|F|<\pi$ on $J \times J$.

(2b) Next suppose $x_{1}$ and $x_{2}$ are such that $\left[x_{1}, x_{2}\right] \subset T_{\delta}$ for some $\delta \in(0, \gamma)$ and that $0<\arg \left(x_{1}\right)-\arg \left(x_{2}\right)<\pi-\delta$. Take $x_{3} \in\left(x_{1}, x_{2}\right)$. Then $0 \geqq F_{1}>-\pi+\delta, F_{2}=0,\left|F_{3}\right|<\delta / 2$, and we have $|F|<\pi$ on $J \times J$.

(2c) Next suppose $x_{1}$ and $x_{2} \in T_{\delta}$ and $\arg \left(x_{1}\right)>\arg \left(x_{2}\right)$, and that $\arg \left(x_{1}\right)-\arg \left(x_{2}\right) \geqq \pi-\delta$ or $\left[x_{1}, x_{2}\right] \nsubseteq T_{\delta}$. Then $x_{1}$ and $x_{2}$ lie on opposite sides of the line $\left\{r e^{i \mu}:-\infty<r<+\infty\right\}$. Let $x_{3}(r)=r e^{i \mu}$ and let $b(r)$ $=\arg \left(x_{3}(r)-x_{1}\right)-\arg \left(x_{2}-x_{3}(r)\right)$. For $r=\xi(\delta), b(r)<(\beta-\delta-\pi)$ $-(\alpha+\delta)<\pi-2 \delta$, while $b(r) \rightarrow \pi$ as $r \rightarrow+\infty$. Hence there exists an $\bar{r}>\xi(\delta)$ such that $b(\bar{r})=\pi-\delta$. Let $x_{3}=x_{3}(\bar{r})$. Observing that $\arg \left(x_{1}\right)$ $-\arg \left(x_{3}\right)<\pi-\delta$ and $\arg \left(x_{3}\right)-\arg \left(x_{2}\right)<\pi-\delta$, we see from (2b) that 
$|F|<\pi$ on $(0,1) \times(0,1) \cup(1,2) \times(1,2)$. Now consider the situation in which $t_{1}<1<t_{2}$. Here $0 \geqq F_{1}>-(\beta-\alpha-2 \delta)>-2 \pi+2 \delta, F_{2}=\pi-\delta$, and $\left|F_{3}\right|<\delta / 2$. Hence $|F|<\pi$ on $J \times J$.

(2d) Finally suppose $x_{1} \in T_{\delta_{1}}-T_{\delta_{2}}, x_{2} \in T_{\delta_{2}}-T_{\delta_{1}}$, where $\arg \left(x_{1}\right)$ $>\arg \left(x_{2}\right)$, and assume $\delta_{1}>\delta_{2}$ with $\xi\left(\delta_{1}\right)<\xi\left(\delta_{2}\right)$ to fix ideas. Let $\left\{\tilde{x}_{3}\right\}$ $=\left\{z: \arg \left(z-x_{1}\right)=\beta-\delta_{1}-\pi\right\} \cap\left\{z: \arg \left(z-x_{2}\right)=\alpha+\delta_{2}-\pi\right\}$. Let $x_{3}(r)$ $=r \tilde{x}_{3}$ for $r \geqq 1$. Then $\left[x_{i}, x_{3}(r)\right] \subset T_{\delta_{i}}$ whenever $r \geqq 1(i=1,2)$. Let $b(r)$ $=\arg \left(x_{3}(r)-x_{1}\right)-\arg \left(x_{2}-x_{3}(r)\right) . \quad b(1)=\beta-\alpha-\delta_{1}-\delta_{2}-\pi \leqq \pi-\delta_{1}-\delta_{2}$, while $b(r) \rightarrow \pi$ as $r \rightarrow+\infty$. Hence there exists an $\bar{r}>1$ such that $b(\bar{r})$ $=\pi-\left(\delta_{1}+\delta_{2}\right) / 2$. Let $x_{3}=x_{3}(\bar{r})$. From $(2 \mathrm{a})-(2 \mathrm{c})$ it follows that $|F|<\pi$ on $(0,1) \times(0,1) \cup(1,2) \times(1,2)$. For $t_{1}<1<t_{2}$ we have $0 \geqq F_{1}>-\left(\beta-\alpha-\delta_{1}-\delta_{2}\right), F_{2}=\pi-\left(\delta_{1}+\delta_{2}\right) / 2$, and $\left|F_{3}\right|<\delta_{1} / 4+\delta_{2} / 4$, whence $|F|<\pi$ on $J \times J$.

Thus $s$ is univalent on $V(\alpha, \beta, \xi)$.

Lemma 4. If $E$ is analytic and $E \rightarrow 0$ over $\bar{F}(\alpha, \beta)$, then $x E^{\prime}(x) \rightarrow 0$ over $\bar{F}(\alpha, \beta)$.

Proof. Let $\epsilon>0$. For each $\delta \in(0,(\beta-\alpha) / 4)$ let $V_{\delta}$ be an element of $\bar{F}(\alpha, \beta)$ such that $|E(x)|<(\epsilon \sin (\delta)) / 2$ on $V_{\delta}$. Let $T\left(\alpha+\delta, \beta-\delta, x_{0}\right) \subset V_{\delta}$, where $x_{0}=r_{0} e^{i \mu}$. Let $T=T\left(\alpha+2 \delta, \beta-2 \delta, x_{0}\right)$. Then for $x \in T$ we have, by the Cauchy integral formula,

$$
\left|x E^{\prime}(x)\right|<\frac{1}{2}(\epsilon \sin (\delta))\left(|x| /\left(\left|x-x_{0}\right| \sin (\delta)\right)\right) .
$$

Let $S=\left\{z:\left|z /\left(z-x_{0}\right)\right|<2\right\}$. Then $\left|x E^{\prime}(x)\right|<\epsilon$ on $S \cap T$, and it is clear that $S \cap T$ contains a sector $T\left(\alpha+2 \delta, \beta-2 \delta, r e^{i \mu}\right)$. Let $T(\epsilon, \delta)$ be such a sector. Then $U\{T(\epsilon, \delta): 0<\delta<(\beta-\alpha) / 4\}$ is an element of $\bar{F}(\alpha, \beta)$ in which $\left|x E^{\prime}(x)\right|<\epsilon$.

Lemma 5. Let $\tilde{M}(x)=x^{1}(\log x)^{n_{1}} \cdots\left(\log _{r} x\right)^{n_{r}}$ and let $\tilde{E}(x) \rightarrow 0$ over $\bar{F}(\alpha, \beta)$. Then $\tilde{M}(1+\tilde{E})$ maps some element of $\bar{F}(\alpha, \beta)$ into the sector $T(\alpha, \beta, 0)$.

Proof. For each $\delta>0$ take $r_{\delta}>0$ to be so large that for $x \in T_{\delta}$ $=T\left(\alpha+\delta, \quad \beta-\delta, \quad r_{\delta} e^{i \mu}\right), \quad|\arg (x)-\arg (\tilde{M}(x)(1+\tilde{E}(x)))|<\delta$. Then $\tilde{M}(1+\tilde{E})$ maps $U T_{\delta}$ into $T(\alpha, \beta, 0)$.

\section{REFERENCES}

1. Walter Strodt, Contributions to the asymptotic theory of ordinary differential equations in the complex domain, Mem. Amer. Math. Soc., No. 13 (1954), 81 pp.

2. - Principal solutions of ordinary differential equations in the complex domain, Mem. Amer. Math. Soc., No. 26 (1957), 107 pp.

3. E. W. Chamberlain, Differential operators approximately of the form $(1-(1 / W(x)) d / d x)^{n}$, Proc. Amer. Math. Soc. 16 (1965), 725-730.

UNIVERSITY OF VERMONT 